\title{
Integrated Bathymetric Data Management - the platform for decision making in the Port of Hamburg
}

Frank KÖSTER, Germany

Head of Hydrographic Surveying Department

Hamburg Port Authority

Ottokarl Büchsenschütz-Nothdurft, The Netherlands

Support \& Training Manager

CARIS BV

Verena Bosselmann-Borsos, Germany

Head of Hydrographic Data Processing

Hamburg Port Authority

Topic: E. easy customer access to data and products (e.g.: webservices; ENC-s)/Tutorial Session

\section{INTRODUCTION}

The Hamburg Port Authority (HPA) has used CARIS tools for bathymetric data management and analysis for more than 10 years. The inclusion of the Engineering Analysis Module (EAM) into the CARIS Bathy DataBASE (BDB) suite together with the professional Oracle based data management and the OGC webservice compliance through CARIS Spatial Fusion Enterprise opens new possibilities for the Hydrographic Survey Department and its customers, as a result of which HPA has now moved to this package.

The CARIS Bathy DataBASE forms the backbone of the bathymetric analysis performed at the HPA and is the platform for several product creation and decision making processes.

Using cleaned multibeam, multichannel and mobile laserscan data and different kinds of harbour maps like ENCs, AutoCAD files or 3D channel models, created within BDB, the Hydrographic Survey Department creates bathymetric models, bathymetric ENCs, all bathymetric objects for paper charts, difference models, profiles and volume computations for the Dredging Department, the Nautical Centre, the pilots, asset managers and other customers (see figure 1).

Additionally customers like the Hydrology Department can either directly access the Bathy DataBASE with the CARIS BASE Manager or can integrate OGC Web Services provided from the CARIS Spatial Fusion Enterprise into their own applications in order to carry out tailored analysis like sediment prediction.

This paper will show how a system like CARIS BDB is integrated into the different workflows within and between the different departments at HPA, showing how to share and jointly use one common data basis over a large and varied user base.

\section{DATA PREPARATION - LOADING DATA INTO THE BATHMETRIC DATABASE}

In order to reduce duplication of effort and chance for error HPA has adapted the standard configuration of the software to HPA specific needs and thus derived a customized workflow which transforms important metadata from the processed and cleaned bathymetric data coming from CARIS HIPS to BDB automatically. When loading the data from HIPS into BDB, metadata information like survey type, survey date or technique of sounding will be automatically mapped and stored as metadata in the surface object, based both on the naming convention of the data as well as existing metadata coming from the HIPS dataset. This is metadata information is then later giving HPA the 
possibility to query and export data based on customers' needs as well as to form decision rules when de-conflicting overlapping data.

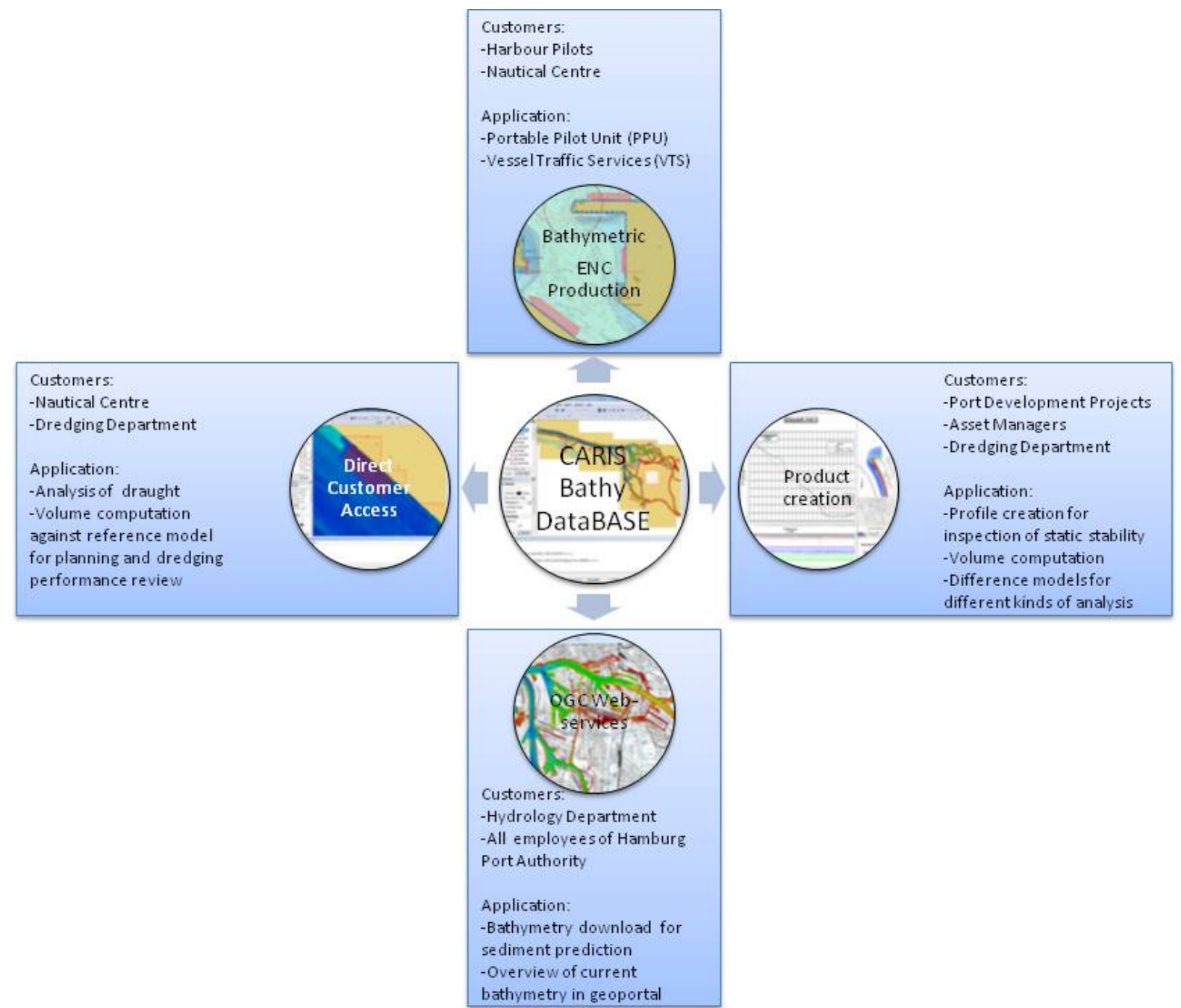

Figure 1: Overview of selected applications of bathymetric data usage and product creation used in the Port of Hamburg. The core of the bathymetric data management is the CARIS Bathy Database. The four main areas of application are bathymetric ENC production (top), Product creation (right), utilisation of OGC Web Services (bottom) and Direct Access to the BDB (left).

\section{DATABASES IN USE}

In order to meet the various products' requirements for up to date survey by survey information on the one side, and seamlessness for the whole port area on the other side, two databases are in constant use as follows:

\section{The "daily" Database}

In highly frequented harbour areas berths are constantly blocked, other survey areas have large extents. For this reason many surveys in the Port of Hamburg are lasting for several days. 
In CARIS HIPS a BASE surface is computed for each day and vessel respectively. It automatically contains all needed metadata and has a resolution from $0,5-1 \mathrm{~m}$. Those surfaces are loaded on a daily basis into the "daily" database containing the metadata and an automatically generated outline. The "Daily Database" is including the data from every survey. Based on the current survey activities and resources, this means that each year around 1500 surfaces will be added.

\section{The "full coverage" Database}

Beside the "daily database" HPA also has a "full coverage" database that is holding the most recent and shoalest data. Every week a surface containing the surveys from the last 7 days is created in the "daily database" which will be combined with the existing most recent data in the "full coverage" database. The result is one surface covering the whole area of the Port of Hamburg saved into the $\mathrm{BDB}$. At each node the most recent and shoalest data is shown.

\section{CREATING PRODUCTS AND SERVICES}

The two bathymetric databases ("daily" and "full coverage") are holding all information in order to derive all services and products the customers of the Hamburg Port Authority demanded.

\section{bENCS}

The bathymetric ENC (bENC) is a product of the "full coverage" database. It is used as high quality data for enhanced port operations and is updated frequently. The bENC is based on the latest hydrographic survey and contains only high-density bathymetry like soundings, depth contours, depth areas and unsurveyed areas.

The team of the Nautical Center utilises the bENCs together with the Port ENCs in order to manage the traffic in the port area (see figure 2).

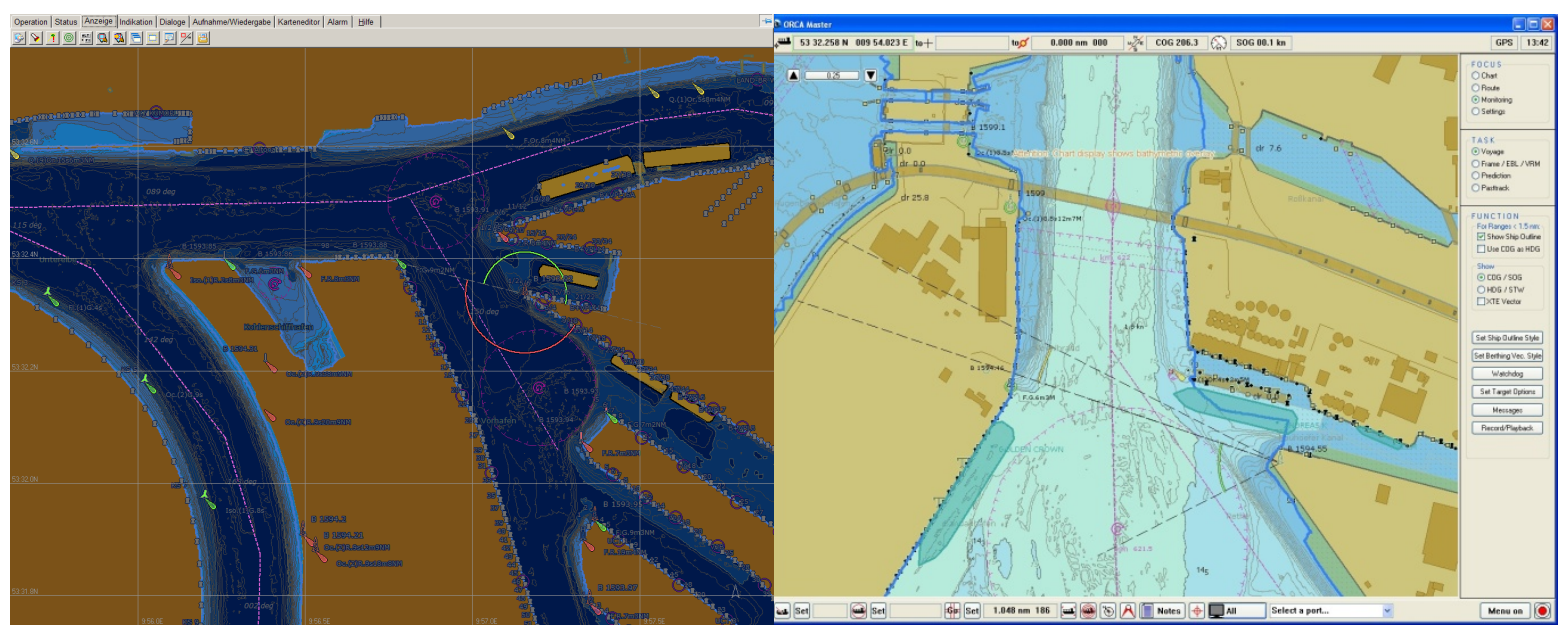

Figure 2: (left) The Vessel Traffic System from SIGNALIS in the Nautical Center of the Port of Hamburg showing the Port ENC superimposed by a bENC in custom colours. (right) The Orca Master/Pilot software of 7Cs is used by the Harbour Pilots in the Port of Hamburg displaying the bENCs and Port ENCs.

The Harbour Pilots will use the bENCs together with the Port ENCs of the Hamburg Port Authority in their Portable Pilot Units (PPUs) (see figure 2). 


\section{Direct Customer Access}

Direct customer access is needed for high performance computation and analysis.

The Nautical Center carries out the analysis of draught for all berths through direct access to the "full coverage" and "daily" databases. The customized metadata workflow enables queries in the "daily" database for newest survey patches. Their outlines are overlaid with the "full coverage" database, where the analysis for the shallowest sounding is carried out (see figure 3).

The Dredging Department carries out volume computations and profile creation directly on the data in the two separate databases "full coverage" and "daily". Mostly volume computations against a reference model's depth are applied for planning or dredging performance review purposes.

\section{OGC Web Services}

The implementation of standardised OGC Web Services with CARIS Spatial Fusion Enterprise using a direct connection to the bathymetric database opens new possibilities for all customers.

The Hydrology Department of the Hamburg Port Authority connects with the sedimentation prediction software SeDiRa of Smile Consult to a combination of three OGC Web Services connected to the "daily" database. The Web Map Service (WMS) graphically shows the outlines of the surface, the Web Feature Service (WFS) delivers all the metadata contained in the bounding polygon of a surface and the Web Coverage Service (WCS) provides the relevant datasets in either Bathymetry Attributed Grid (BAG) format or ASCII XYZ files.

All employees of the Hamburg Port Authority can use the GeoPortal realised by AED-SICAD. The GeoPortal includes a WMS service accessing the "full coverage" database (see figure 3).

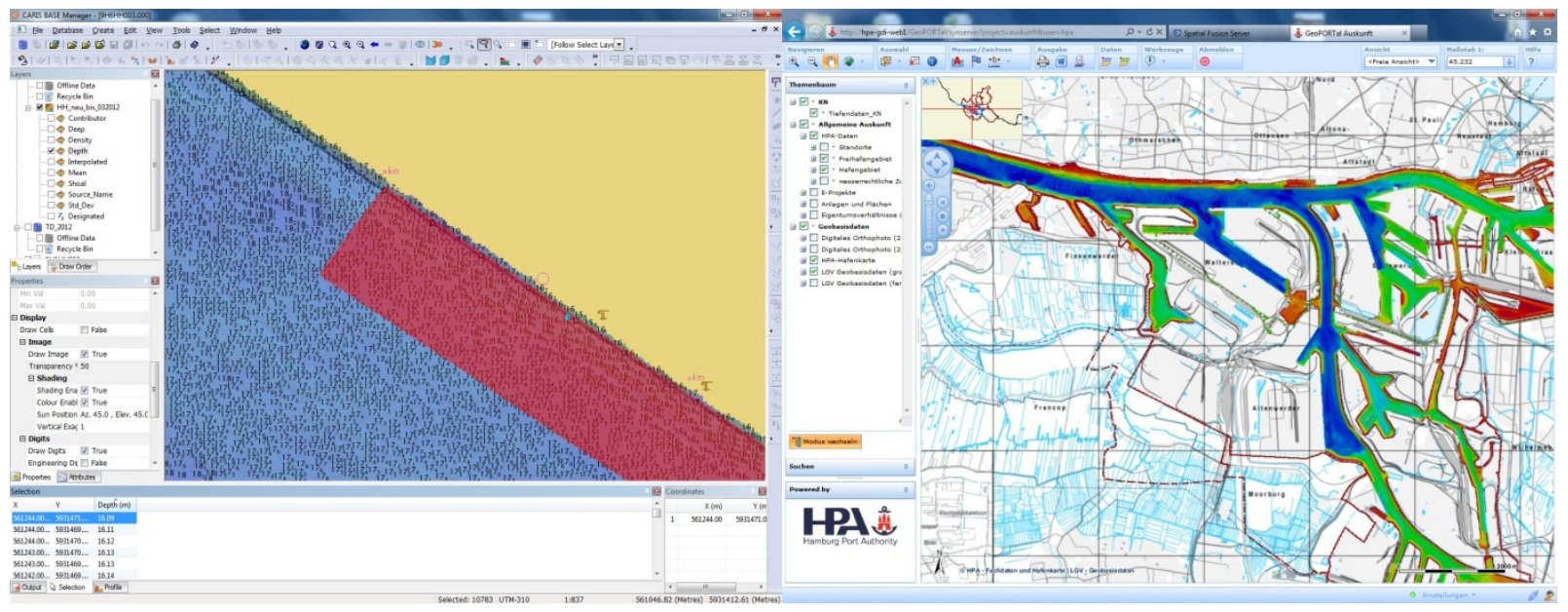

Figure 3: (left) In the CARIS Bathy Database Manager the analysis for the least depth at a berth is done by selecting the extent of the berth and searching for the shoalest sounding. (right) The GeoPortal includes a colour coded bathymetry layer covering the full extent of the Port of Hamburg and served from the SFE .

\section{Paper Product Creation}

Being able to create products from bathymetric data according to customers' needs is one core business of the Hydrographic Department of the Hamburg Port Authority. 
With the addition of the Engineering Analysis Module into the Bathy DataBASE it is now possible to not only compute difference surfaces between two surfaces (see figure 5) but also of surfaces against a reference model. The difference surface can be customized with different colour shadings and if needed soundings and contours can be created. Another frequently requested product is the profile. Profiles are computed for surfaces together with the reference depth in BDB, and are visualised in Golden Software Grapher (see figure 4).

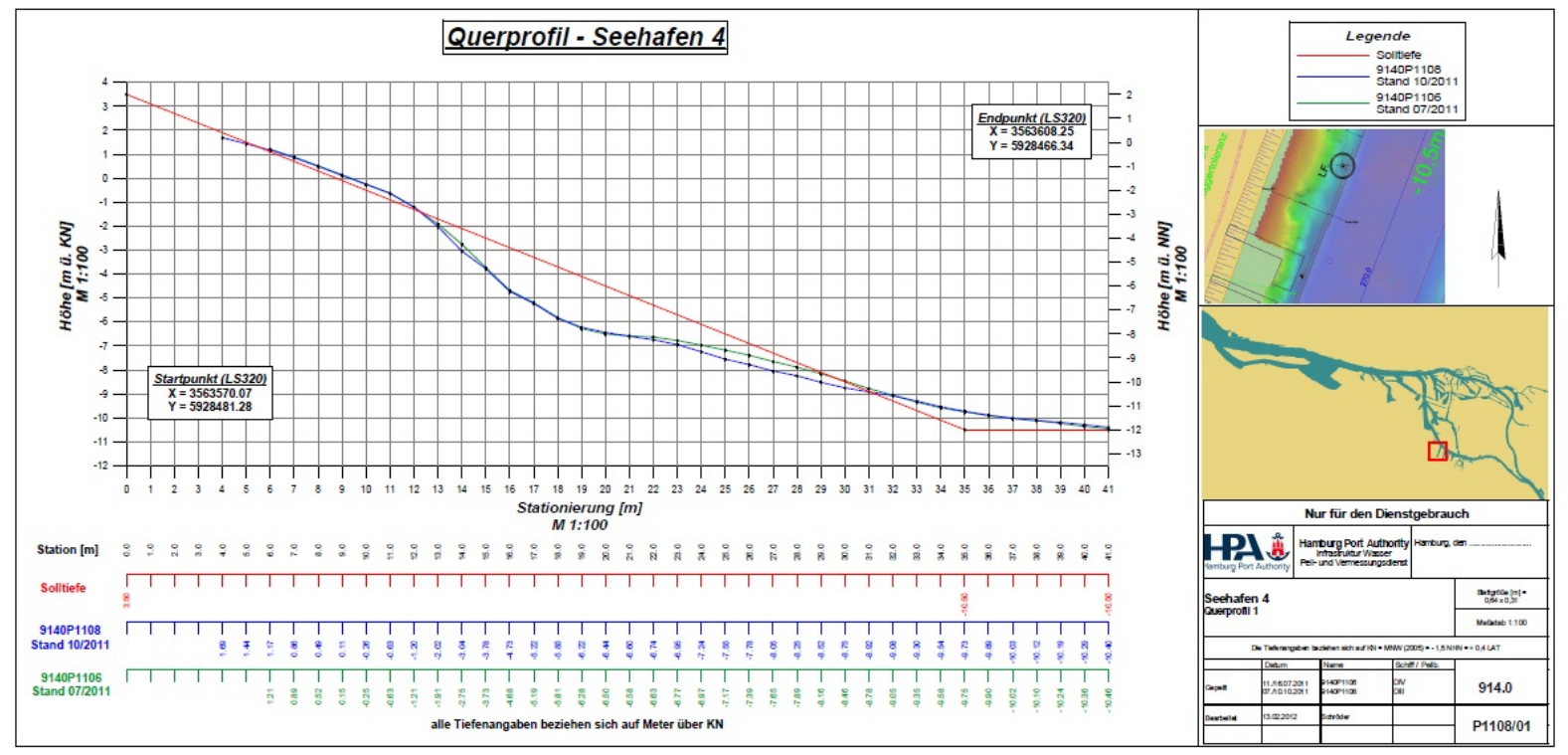

Figure 4: Profile at the "Seehafen 4" in Hamburg-Harburg. The red line depicts the reference model. The green and blue lines show survey data from two bathymetric surveys.

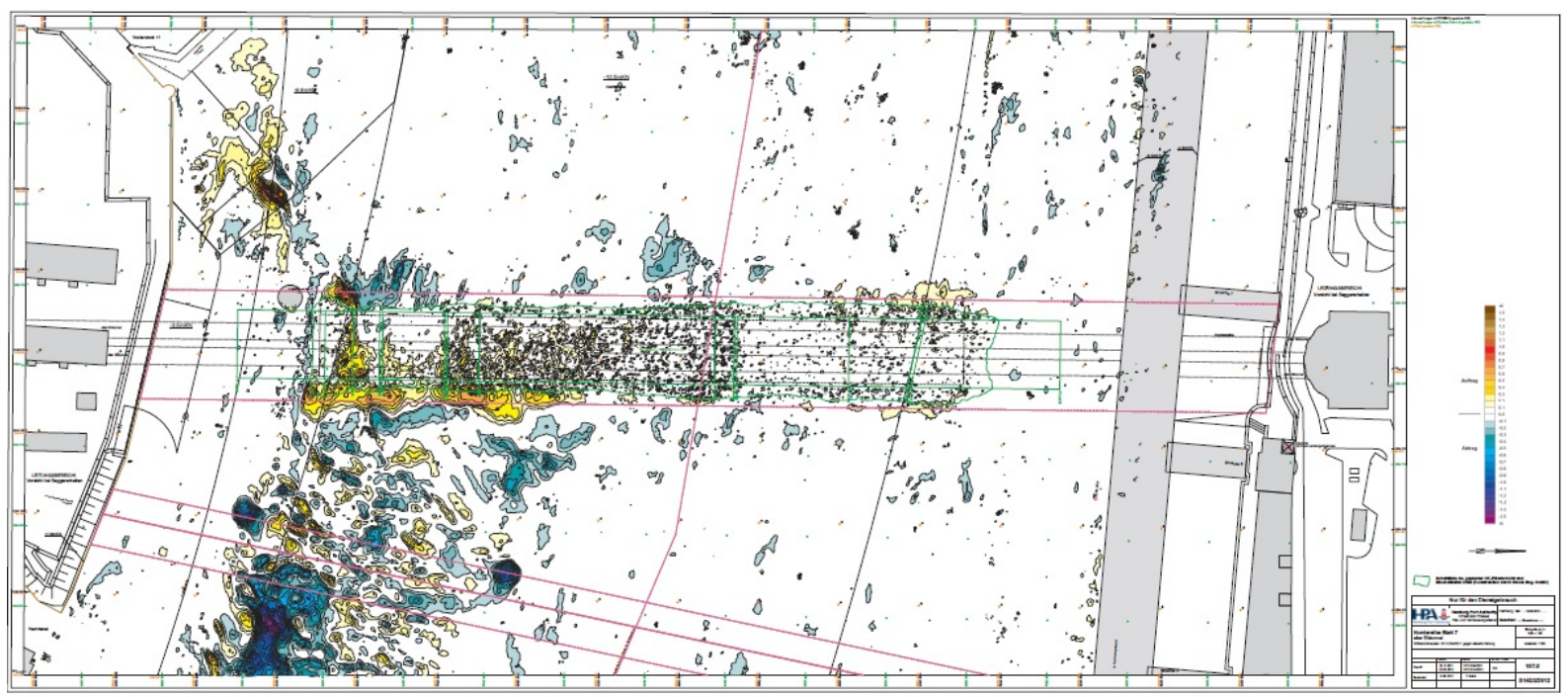

Figure 5: Difference model map compiled in CARIS HPD Paper Chart Editor. The depth contours and depth areas have been computed in CARIS BDB. This map shows a difference between two surveys with an interval of 6 months. 


\section{CONCLUSION}

The combination of the CARIS tools Bathy DataBASE and Spatial Fusion Enterprise provides an abundance of possibilities for HPA and their customers to maintain, access, produce and analyse bathymetric data in an efficient way. The application of open or standard formats like the OGC web standards or the bENC standard enable other users and software packages to utilise the bathymetric data for various different use cases. As a result these departments within HPA that require bathymetry for their planning and decision making can all draw on one common data source.

The addition of the Engineering Analysis Module to the Bathy DataBASE has expanded the creation of products to volume computation, profiles and difference models and reduced the need for the Hydrographic Department to keep another extra software for that specific purpose.

Further developments of the standard applications, which HPA will receive automatically as a result of a maintenance contract, will be reviewed when they become available, to see how they can help to both optimize the workflows further as well as in providing new products to HPA's customers.

\section{BIOGRAPHIES}

Frank KÖSTER

2003 Dipl.-Ing. (FH) Geomatics, Surveying and Hydrography (CAT A) at HAW Hamburg

2004-2010 Head of Hydrographic Data Processing at Hamburg Port Authority

2006 Master of Science in Hydrography at HafenCity Unversität Hamburg

Since 2010 Head of Hydrographic Department at Hamburg Port Authority

\section{Verena BOSSELMANN-BORSOS}

2002 Dipl.-Ing. (FH) Geomatics, Surveying at HAW Hamburg

2002 - 2004 Quality Assurance for ENC Production at SevenCs

2004 - 2007 Head of Quality Assurance for ENC Production at SevenCs

2007 Data Analyst at HSA Systems Pty LTD, Australia

2007 - 2008 Data Analyst at New Zealand Hydrographic Office

2008 - 2010 Production Manager at New Zealand Hydrographic Office

Since 2011 Head of Hydrographic Data Processing at Hamburg Port Authority

Ottokarl BÜCHSENSCHÜTZ-NOTHDURFT, Support \& Training Manager at CARIS BV. With a degree of Geodesy and Survey Engineering from the University of Hannover and a CAT A (Academic) from the University of New Brunswick, he has joined CARIS BV in late 2002 as a Hydrographic Engineer in the Support \& Training Department. Since then he has moved on through the position of Support \& Training Coordinator to become the manager of this team in 2006. He is still actively providing training and consultancy in the products CARIS HIPS and SIPS and CARIS Bathy DataBASE amongst others. 
CONTACT DETAILS (of corresponding author only)

Frank KÖSTER

Hamburg Port Authority

Neuer Wandrahm 4

20457 Hamburg

GERMANY

Tel.: +49 40 / 42847-2385

Fax: +4940 / 42847-2048

Email: frank.koester@hpa.hamburg.de

Web site: www.hamburg-port-authority.de

LinkedIn account: \%

Facebook account: \%

Twitter account: \% 